\title{
Solutions to Time-Fractional Diffusion-Wave Equation in Cylindrical Coordinates
}

\author{
Y. Z. Povstenko ${ }^{1,2}$ \\ ${ }^{1}$ Institute of Mathematics and Computer Science, Jan Długosz University, 42200 Częstochowa, Poland \\ ${ }^{2}$ Department of Computer Science, European University of Informatics and Economics (EWSIE), \\ 03741 Warsaw, Poland
}

Correspondence should be addressed to Y. Z. Povstenko, j.povstenko@ajd.czest.pl

Received 8 December 2010; Accepted 6 February 2011

Academic Editor: J. J. Trujillo

Copyright @ 2011 Y. Z. Povstenko. This is an open access article distributed under the Creative Commons Attribution License, which permits unrestricted use, distribution, and reproduction in any medium, provided the original work is properly cited.

Nonaxisymmetric solutions to time-fractional diffusion-wave equation with a source term in cylindrical coordinates are obtained for an infinite medium. The solutions are found using the Laplace transform with respect to time $t$, the Hankel transform with respect to the radial coordinate $r$, the finite Fourier transform with respect to the angular coordinate $\varphi$, and the exponential Fourier transform with respect to the spatial coordinate $z$. Numerical results are illustrated graphically.

\section{Introduction}

The time-fractional diffusion-wave equation

$$
\frac{\partial^{\alpha} u}{\partial t^{\alpha}}=a \Delta u
$$

is a mathematical model of important physical phenomena ranging from amorphous, colloid, glassy, and porous materials through fractals, percolation clusters, random, and disordered media to comb structures, dielectrics and semiconductors, polymers, and biological systems (see [1-10] and references therein).

The fundamental solution for the fractional diffusion-wave equation in one spacedimension was obtained by Mainardi [11]. Wyss [12] obtained the solutions to the Cauchy problem in terms of $H$-functions using the Mellin transform. Schneider and Wyss [13] converted the diffusion-wave equation with appropriate initial conditions into the integrodifferential equation and found the corresponding Green functions in terms of Fox 


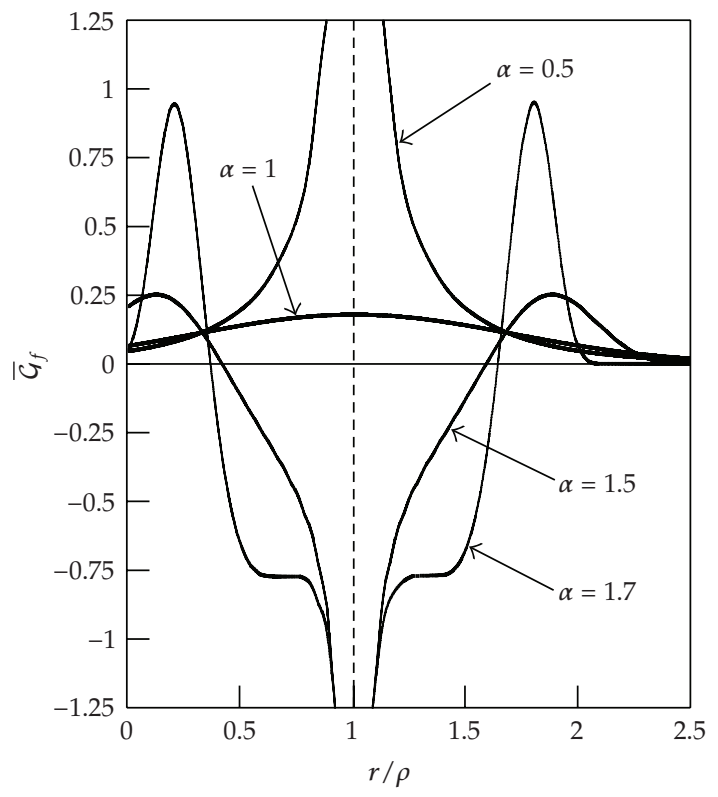

Figure 1: Dependence of nondimensional fundamental solution $\mathcal{G}_{f}(r, \varphi, z, \rho, \phi, \zeta, t)$ on the radial coordinate $r$ for $\phi=0, \zeta=0, z=0, \varphi=0$, and $\kappa=0.5$.

functions. Fujita [14] treated integrodifferential equation which interpolates the diffusion equation and the wave equation. Hanyga [15] studied Green functions and propagator functions in one, two, and three dimensions.

Previously, in studies concerning time-fractional diffusion-wave equation in cylindrical coordinates, only one or two spatial coordinates have been considered [16-27]. In this paper, we investigate solutions to (1.1) in an infinite medium in cylindrical coordinates in the case of three spatial coordinates $r, \varphi$, and $z$.

\section{Statement of the Problem}

Consider the time-fractional diffusion-wave equation with a source term in cylindrical coordinates

$$
\begin{array}{r}
\frac{\partial^{\alpha} u}{\partial t^{\alpha}}=a\left(\frac{\partial^{2} u}{\partial r^{2}}+\frac{1}{r} \frac{\partial u}{\partial r}+\frac{1}{r^{2}} \frac{\partial^{2} u}{\partial \varphi^{2}}+\frac{\partial^{2} u}{\partial z^{2}}\right)+Q(r, \varphi, z, t), \quad 0 \leq r<\infty, \\
0 \leq \varphi \leq 2 \pi, \quad-\infty<z<\infty, \quad 0<t<\infty, \quad 0<\alpha \leq 2 .
\end{array}
$$

The initial conditions are prescribed:

$$
\begin{gathered}
t=0: u=f(r, \varphi, z), \quad 0<\alpha \leq 2, \\
t=0: \frac{\partial u}{\partial t}=F(r, \varphi, z), \quad 1<\alpha \leq 2 .
\end{gathered}
$$




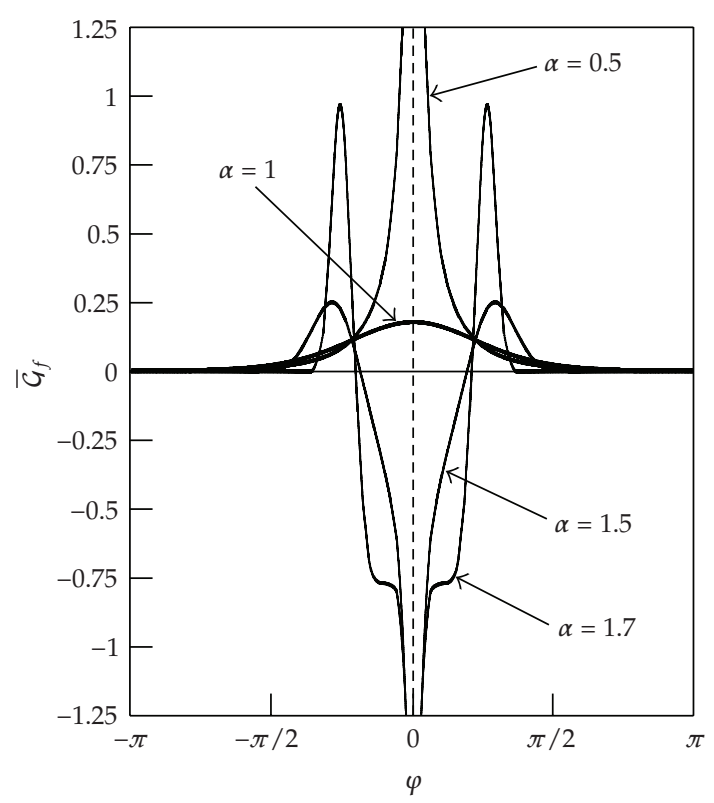

Figure 2: Dependence of nondimensional fundamental solution $\mathcal{G}_{f}(r, \varphi, z, \rho, \phi, \zeta, t)$ on the angular coordinate $\varphi$ for $\phi=0, \zeta=0, r=\rho, z=0$, and $\kappa=0.5$.

In (2.1), we use the Caputo fractional derivative [28-30]

$$
\frac{\mathrm{d}^{\alpha} u(t)}{\mathrm{d} t^{\alpha}}= \begin{cases}\frac{1}{\Gamma(n-\alpha)} \int_{0}^{t}(t-\tau)^{n-\alpha-1} \frac{\mathrm{d}^{n} u(\tau)}{\mathrm{d} \tau^{n}} \mathrm{~d} \tau, & n-1<\alpha<n, \\ \frac{\mathrm{d}^{n}}{\mathrm{~d} t^{n}} u(t), & \alpha=n,\end{cases}
$$

where $\Gamma(\alpha)$ is the gamma function. For its Laplace transform rule, the Caputo fractional derivative requires the knowledge of the initial values of the function $u(t)$ and its integer derivatives of order $k=1,2, \ldots, n-1$ :

$$
\mathcal{L}\left\{\frac{\mathrm{d}^{\alpha} u(t)}{\mathrm{d} t^{\alpha}}\right\}=s^{\alpha} \mathcal{L}\{u(t)\}-\sum_{k=0}^{n-1} u^{(k)}\left(0^{+}\right) s^{\alpha-1-k}, \quad n-1<\alpha<n,
$$

where $s$ is the transform variable. 


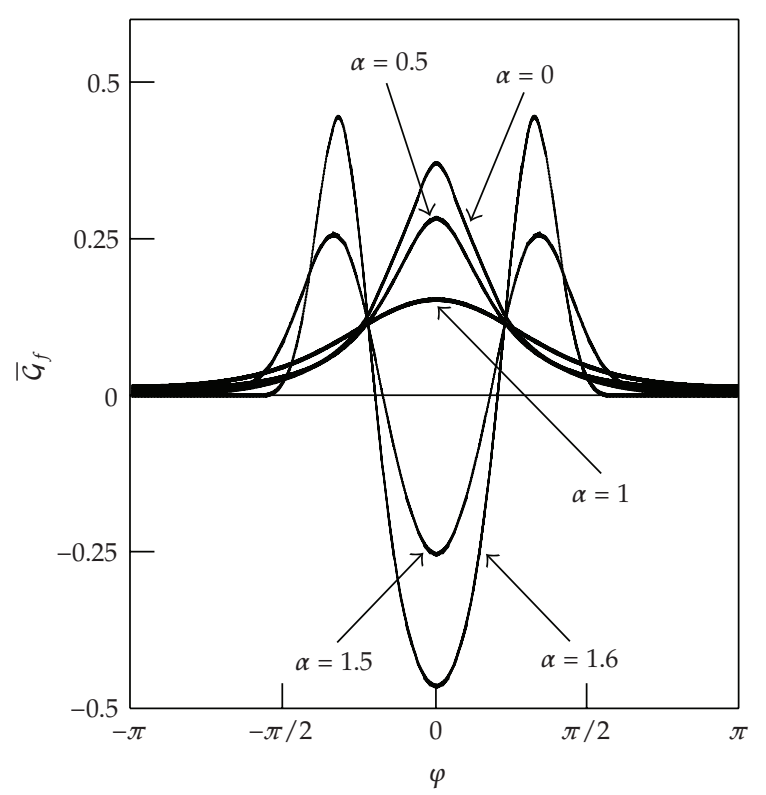

Figure 3: Dependence of nondimensional fundamental solution $\mathcal{G}_{f}(r, \varphi, z, \rho, \phi, \zeta, t)$ on the angular coordinate $\varphi$ for $\phi=0, \zeta=0, r=0.6 \rho, z=0$, and $\kappa=0.5$. form:

The solution to the initial-value problem (2.1)-(2.2) can be written in the following

$$
\begin{aligned}
u= & \int_{-\infty}^{\infty} \int_{0}^{2 \pi} \int_{0}^{\infty} f(\rho, \phi, \zeta) \mathcal{G}_{f}(r, \varphi, z, \rho, \phi, \zeta, t) \rho \mathrm{d} \rho \mathrm{d} \phi \mathrm{d} \zeta \\
& +\int_{-\infty}^{\infty} \int_{0}^{2 \pi} \int_{0}^{\infty} F(\rho, \phi, \zeta) \mathcal{G}_{F}(r, \varphi, z, \rho, \phi, \zeta, t) \rho \mathrm{d} \rho \mathrm{d} \phi \mathrm{d} \zeta \\
& +\int_{0}^{t} \int_{-\infty}^{\infty} \int_{0}^{2 \pi} \int_{0}^{\infty} Q(\rho, \phi, \zeta, \tau) \mathcal{G}_{Q}(r, \varphi, z, \rho, \phi, \zeta, t-\tau) \rho \mathrm{d} \rho \mathrm{d} \phi \mathrm{d} \zeta \mathrm{d} \tau .
\end{aligned}
$$

Now, we investigate the fundamental solutions $\mathcal{G}_{f}(r, \varphi, z, \rho, \phi, \zeta, t), \mathcal{G}_{F}(r, \varphi, z, \rho, \phi, \zeta, t)$, and $\mathcal{G}_{Q}(r, \varphi, z, \rho, \phi, \zeta, t)$.

\section{Fundamental Solution to the First Cauchy Problem}

In the case of the first Cauchy problem, the initial value of a sought-for function is prescribed. Hence, 


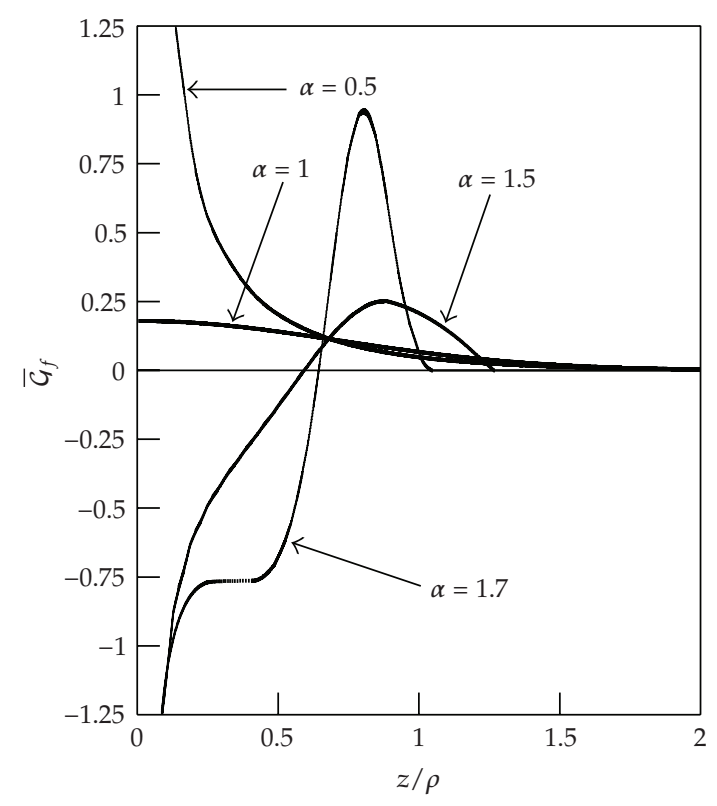

Figure 4: Dependence of nondimensional fundamental solution $\mathcal{G}_{f}(r, \varphi, z, \rho, \phi, \zeta, t)$ on the spatial coordinate $z$ for $\phi=0, \zeta=0, r=\rho, \varphi=0$, and $\kappa=0.5$.

$$
\begin{gathered}
\frac{\partial^{\alpha} \mathcal{G}_{f}}{\partial t^{\alpha}}=a\left(\frac{\partial^{2} \mathcal{G}_{f}}{\partial r^{2}}+\frac{1}{r} \frac{\partial \mathcal{G}_{f}}{\partial r}+\frac{1}{r^{2}} \frac{\partial^{2} \mathcal{G}_{f}}{\partial \varphi^{2}}+\frac{\partial^{2} \mathcal{G}_{f}}{\partial z^{2}}\right), \quad 0 \leq r<\infty, \\
0 \leq \varphi \leq 2 \pi, \quad-\infty<z<\infty, \quad 0<t<\infty, \\
t=0: \quad \mathcal{G}_{f}=f_{0} \frac{\delta(r-\rho)}{r} \delta(\varphi-\phi) \delta(z-\zeta), \quad 0<\alpha \leq 2, \\
t=0: \quad \frac{\partial \mathcal{G}_{f}}{\partial t}=0, \quad 1<\alpha \leq 2 .
\end{gathered}
$$

The two-dimensional Dirac delta function $\delta(x) \delta(y)$ after passing to the polar coordinates takes the form $(1 / 2 \pi r) \delta_{+}(r)$, but for the sake of simplicity, we have omitted the multiplier $2 \pi$ in the solution (2.5) as well as $1 / 2 \pi$ in (3.2). In the initial condition (3.2), we have introduced the constant multiplier $f_{0}$ to obtain the nondimensional quantity $\overline{\mathcal{G}}_{f}$ (see (3.10)).

The solution is found using the Laplace transform with respect to time $t$, the Hankel transform with respect to the radial coordinate $r$, the finite Fourier transform with respect to the angular coordinate $\varphi$, and the exponential Fourier transform with respect to the spatial coordinate $z$. In the transforms domain we get

$$
\mathcal{G}_{f}^{*}=\frac{1}{\sqrt{2 \pi}} \mathrm{e}^{i \eta \zeta} \cos [n(\varphi-\phi)] J_{n}(\rho \xi) \frac{s^{\alpha-1}}{s^{\alpha}+a\left(\xi^{2}+\eta^{2}\right)}
$$




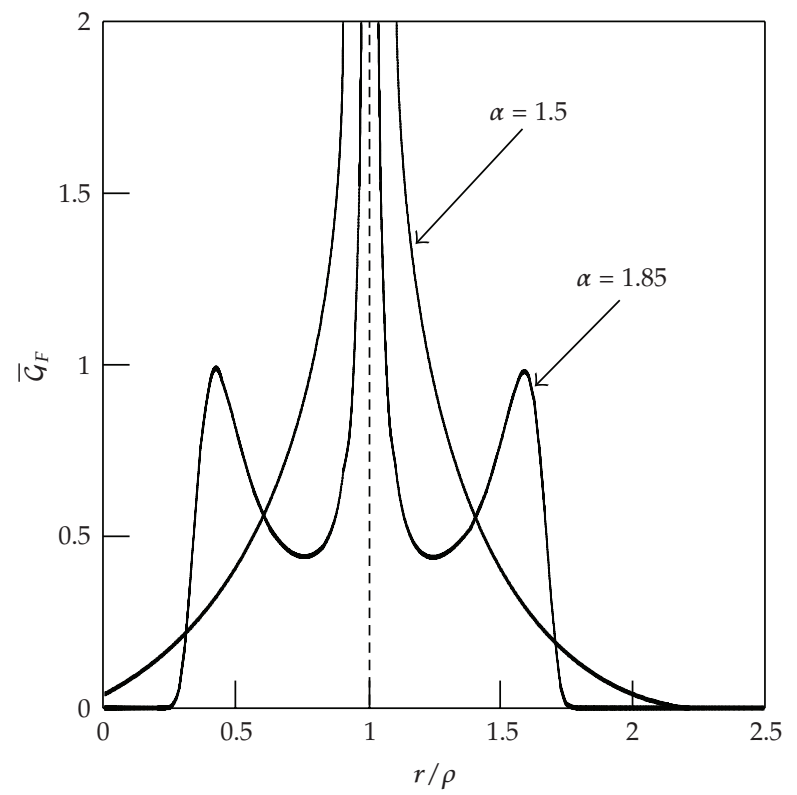

Figure 5: Dependence of nondimensional fundamental solution $\mathcal{G}_{F}(r, \varphi, z, \rho, \phi, \zeta, t)$ on the radial coordinate $r$ for $\phi=0, \zeta=0, z=0, \varphi=0$, and $\kappa=0.5$.

where $J_{n}(r)$ is the Bessel function of the first kind of order $n$, the asterisk indicates the transforms, $s$ is the Laplace transform variable, $\xi$ is the Hankel transform variable, $\eta$ is exponential Fourier transform variable, and the integer $n$ is finite Fourier transform variable.

Inversion of integral transforms gives

$$
\begin{aligned}
\mathcal{G}_{f}(r, \varphi, z, \rho, \phi, \zeta, t)=\frac{1}{2 \pi^{2}} \sum_{n=0}^{\infty} \int_{-\infty}^{\infty} \int_{0}^{\infty} & E_{\alpha}\left[-a\left(\xi^{2}+\eta^{2}\right) t^{\alpha}\right] \\
& \times \cos [n(\varphi-\phi)] \cos [(z-\zeta) \eta] J_{n}(\rho \xi) J_{n}(r \xi) \xi \mathrm{d} \xi \mathrm{d} \eta,
\end{aligned}
$$

where the prime denotes that the term corresponding to $n=0$ in the sum should be multiplied by $1 / 2$. In (3.5), $E_{\alpha}(z)$ is the Mittag-Leffler function [28-31]

$$
E_{\alpha}(z)=\sum_{n=0}^{\infty} \frac{z^{n}}{\Gamma(\alpha n+1)}, \quad \alpha>0, z \in C
$$

The essential role of the Mittag-Leffler function in fractional calculus results from the following formula for the inverse Laplace transform [28-30]:

$$
\mathcal{L}^{-1}\left\{\frac{s^{\alpha-1}}{S^{\alpha}+b}\right\}=E_{\alpha}\left(-b t^{\alpha}\right) .
$$




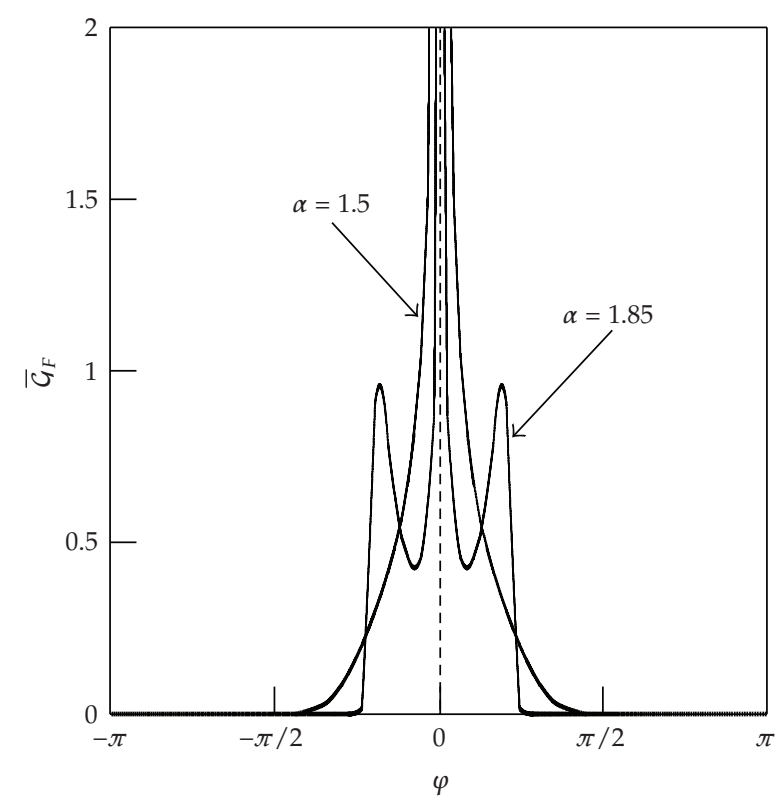

Figure 6: Dependence of nondimensional fundamental solution $\mathcal{G}_{F}(r, \varphi, z, \rho, \phi, \zeta, t)$ on the angular coordinate $\varphi$ for $\phi=0, \zeta=0, r=\rho, z=0$, and $\kappa=0.5$.

If the solution does not depend on the coordinate $z$, then

$$
\mathcal{G}_{f}(r, \varphi, \rho, \phi, t)=\frac{1}{\pi} \sum_{n=0}^{\infty} \int_{0}^{\infty} E_{\alpha}\left(-a \xi^{2} t^{\alpha}\right) \cos [n(\varphi-\phi)] J_{n}(\rho \xi) J_{n}(r \xi) \xi \mathrm{d} \xi
$$

The fundamental solution (3.8) was considered in [25] for $0 \leq \alpha \leq 1$.

In the case when the solution does not also depend on the angular coordinate $\varphi$, we get [17]

$$
\mathcal{G}_{f}(r, \rho, t)=\int_{0}^{\infty} E_{\alpha}\left(-a \xi^{2} t^{\alpha}\right) J_{0}(\rho \xi) J_{0}(r \xi) \xi \mathrm{d} \xi
$$

Dependence of fundamental solution (3.5) on the coordinates $r, \varphi$, and $z$ is presented in Figures 1, 2, 3, and 4.

In calculations, we have introduced nondimensional quantities:

$$
\overline{\mathcal{G}}_{f}=\frac{\rho^{3}}{f_{0}} \mathcal{G}_{f}, \quad \kappa=\frac{\sqrt{a} t^{\alpha / 2}}{\rho}
$$




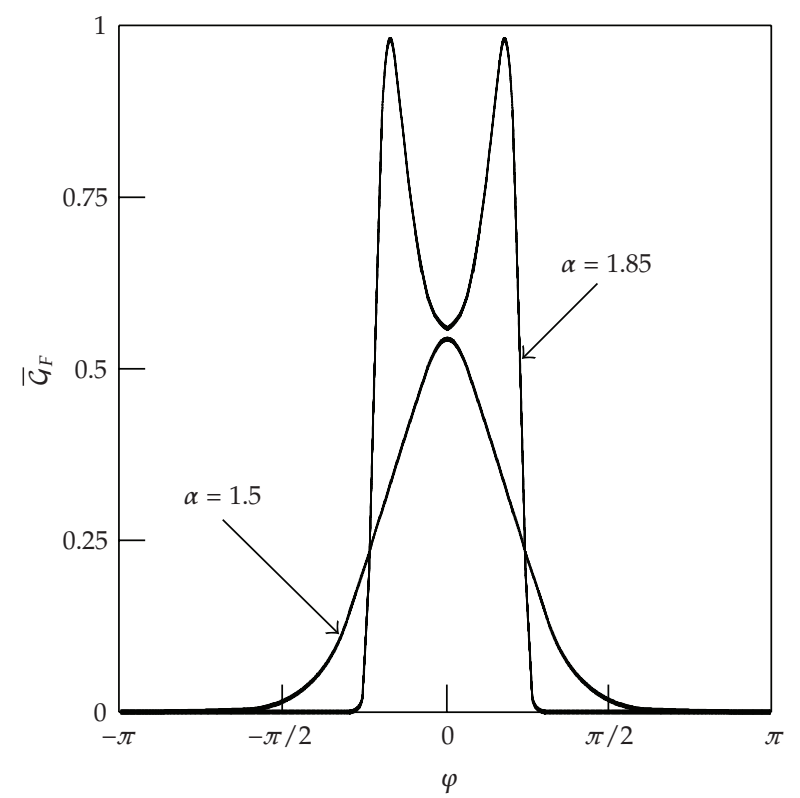

Figure 7: Dependence of nondimensional fundamental solution $\mathcal{G}_{F}(r, \varphi, z, \rho, \phi, \zeta, t)$ on the angular coordinate $\varphi$ for $\phi=0, \zeta=0, r=0.6 \rho, z=0$, and $\kappa=0.5$.

\section{Fundamental Solution to the Second Cauchy Problem}

In the case of the second Cauchy problem, the initial value of the time derivative of a soughtfor function is prescribed, and for the corresponding fundamental solution we have

$$
\begin{aligned}
& \frac{\partial^{\alpha} \mathcal{G}_{F}}{\partial t^{\alpha}}=a\left(\frac{\partial^{2} \mathcal{G}_{F}}{\partial r^{2}}+\frac{1}{r} \frac{\partial \mathcal{G}_{F}}{\partial r}+\frac{1}{r^{2}} \frac{\partial^{2} \mathcal{G}_{F}}{\partial \varphi^{2}}+\frac{\partial^{2} \mathcal{G}_{F}}{\partial z^{2}}\right), \quad 0 \leq r<\infty, \\
& 0 \leq \varphi \leq 2 \pi, \quad-\infty<z<\infty, \quad 0<t<\infty, \\
& t=0: \quad \mathcal{G}_{F}=0, \quad 1<\alpha \leq 2, \\
& t=0: \quad \frac{\partial \mathcal{G}_{F}}{\partial t}=F_{0} \frac{\delta(r-\rho)}{r} \delta(\varphi-\phi) \delta(z-\zeta), \quad 1<\alpha \leq 2 .
\end{aligned}
$$

In this instance, the fundamental solution is expressed as

$$
\begin{aligned}
\mathcal{G}_{F}(r, \varphi, z, \rho, \phi, \zeta, t)=\frac{1}{2 \pi^{2}} \sum_{n=0}^{\infty} \int_{-\infty}^{\infty} \int_{0}^{\infty} & t E_{\alpha, 2}\left[-a\left(\xi^{2}+\eta^{2}\right) t^{\alpha}\right] \\
& \times \cos [n(\varphi-\phi)] \cos [(z-\zeta) \eta] J_{n}(\rho \xi) J_{n}(r \xi) \xi \mathrm{d} \xi \mathrm{d} \eta,
\end{aligned}
$$




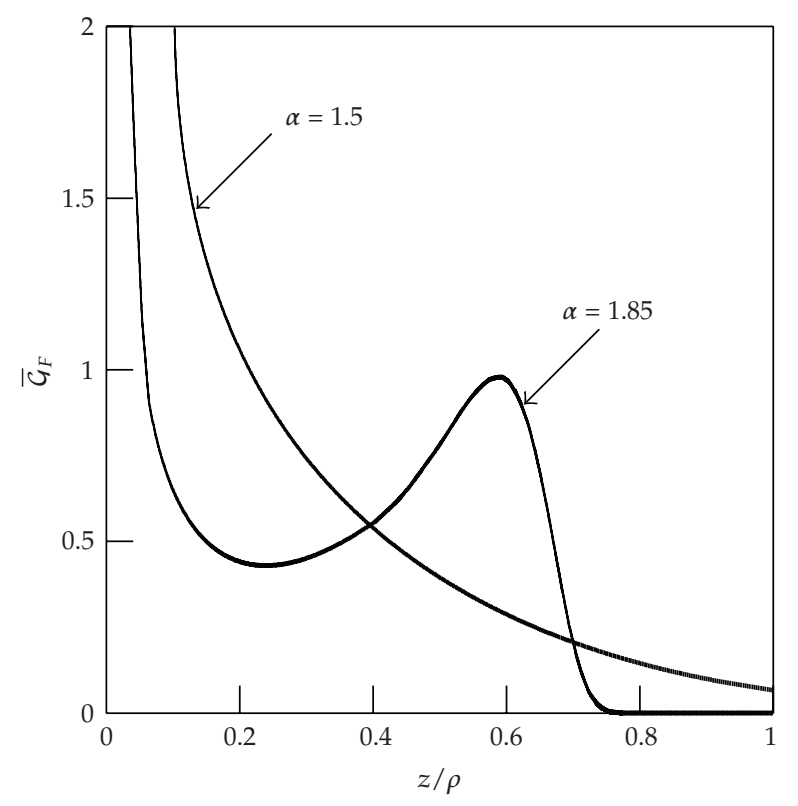

Figure 8: Dependence of nondimensional fundamental solution $\mathcal{G}_{F}(r, \varphi, z, \rho, \phi, \zeta, t)$ on the spatial coordinate $z$ for $\phi=0, \zeta=0, r=\rho, \varphi=0$, and $\mathcal{\kappa}=0.5$.

where $E_{\alpha, \beta}(z)$ is the generalized Mittag-Leffler function in two parameters $\alpha$ and $\beta$ [29-32]:

$$
E_{\alpha, \beta}(z)=\sum_{n=0}^{\infty} \frac{z^{n}}{\Gamma(\alpha n+\beta)}, \quad \alpha>0, \beta>0, z \in C
$$

We have used the following formula for the inverse Laplace transform [29-31]

$$
\mathcal{L}^{-1}\left\{\frac{s^{\alpha-\beta}}{s^{\alpha}+b}\right\}=t^{\beta-1} E_{\alpha, \beta}\left(-b t^{\alpha}\right)
$$

It is evident that (3.7) is the particular case of (4.4) corresponding to $\beta=1$.

If the solution does not depend on the coordinate $z$, then

$$
\mathcal{G}_{F}(r, \varphi, \rho, \phi, t)=\frac{1}{\pi} \sum_{n=0}^{\infty} \int_{0}^{\infty} t E_{\alpha, 2}\left(-a \xi^{2} t^{\alpha}\right) \cos [n(\varphi-\phi)] J_{n}(\rho \xi) J_{n}(r \xi) \xi \mathrm{d} \xi
$$

In the case of axial symmetry [17],

$$
\mathcal{G}_{F}(r, \rho, t)=\int_{0}^{\infty} t E_{\alpha, 2}\left(-a \xi^{2} t^{\alpha}\right) J_{0}(\rho \xi) J_{0}(r \xi) \xi \mathrm{d} \xi
$$




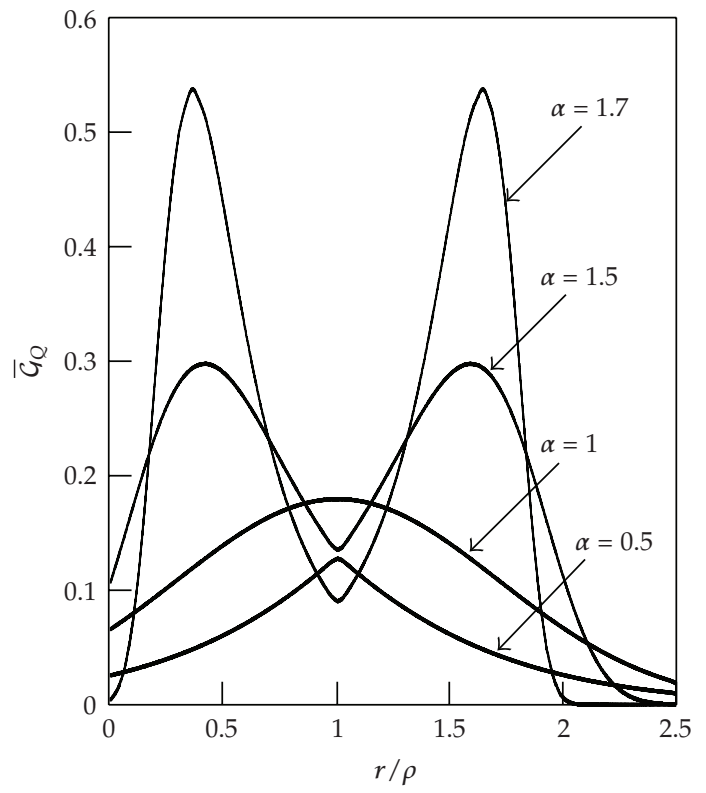

Figure 9: Dependence of nondimensional fundamental solution $\mathcal{G}_{Q}(r, \varphi, z, \rho, \phi, \zeta, t)$ on the radial coordinate $r$ for $\phi=0, \zeta=0, z=0, \varphi=0$, and $\kappa=0.5$.

Figures 5, 6, 7, and 8 show dependence of fundamental solution (4.2) on coordinates $r, \varphi$, and $z$, where

$$
\overline{\mathcal{G}}_{F}=\frac{\rho^{3}}{t F_{0}} \mathcal{G}_{F}
$$

and the nondimensional quantity $\kappa$ is the same as in (3.10).

\section{Fundamental Solution to the Source Problem}

Consider the time-fractional diffusion-wave equation with a source term under zero initial conditions:

$$
\begin{gathered}
\frac{\partial^{\alpha} \mathcal{G}_{Q}}{\partial t^{\alpha}}=a\left(\frac{\partial^{2} \mathcal{G}_{Q}}{\partial r^{2}}+\frac{1}{r} \frac{\partial \mathcal{G}_{Q}}{\partial r}+\frac{1}{r^{2}} \frac{\partial^{2} \mathcal{G}_{Q}}{\partial \varphi^{2}}+\frac{\partial^{2} \mathcal{G}_{Q}}{\partial z^{2}}\right) \\
+Q_{0} \frac{\delta(r-\rho)}{r} \delta(\varphi-\phi) \delta(z-\zeta) \delta_{+}(t), \quad 0 \leq r<\infty, \\
0 \leq \varphi \leq 2 \pi, \quad-\infty<z<\infty, \quad 0<t<\infty, \\
t=0: \mathcal{G}_{Q}=0, \quad 0<\alpha \leq 2, \\
t=0: \frac{\partial \mathcal{G}_{Q}}{\partial t}=0, \quad 1<\alpha \leq 2 .
\end{gathered}
$$




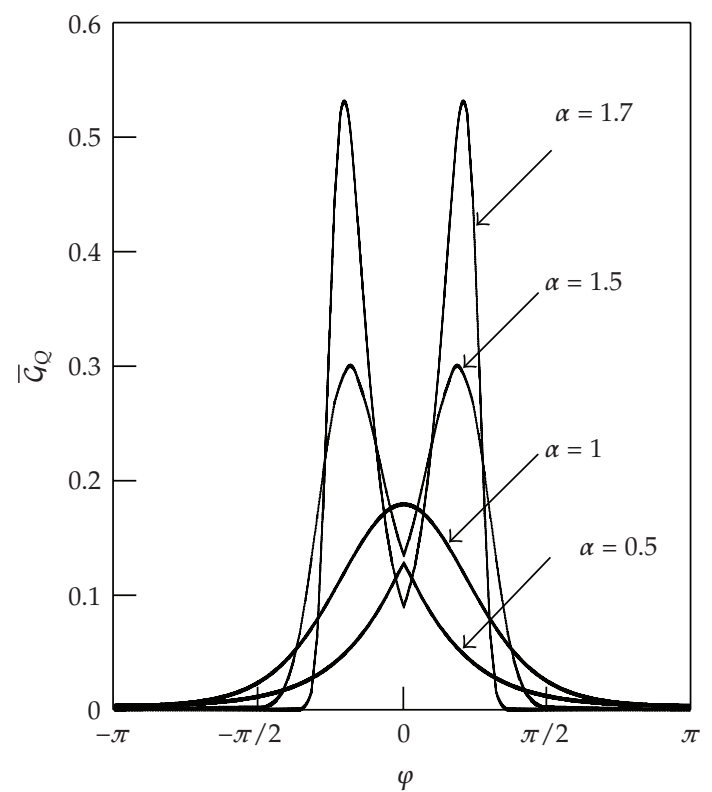

Figure 10: Dependence of nondimensional fundamental solution $\mathcal{G}_{Q}(r, \varphi, z, \rho, \phi, \zeta, t)$ on the angular coordinate $\varphi$ for $\phi=0, \zeta=0, r=\rho, z=0$, and $\kappa=0.5$.

The solution is obtained using the integral transform technique and reads

$$
\begin{aligned}
\mathcal{G}_{Q}(r, \varphi, z, \rho, \phi, \zeta, t)=\frac{1}{2 \pi^{2}} \sum_{n=0}^{\infty} \int_{-\infty}^{\infty} \int_{0}^{\infty} & t^{\alpha-1} E_{\alpha, \alpha}\left[-a\left(\xi^{2}+\eta^{2}\right) t^{\alpha}\right] \\
& \times \cos [n(\varphi-\phi)] \cos [(z-\zeta) \eta] J_{n}(\rho \xi) J_{n}(r \xi) \xi \mathrm{d} \xi \mathrm{d} \eta .
\end{aligned}
$$

If dependence of solution on the coordinate $z$ is not taken into account, then

$$
\mathcal{G}_{Q}(r, \varphi, \rho, \phi, t)=\frac{1}{\pi} \sum_{n=0}^{\infty} \int_{0}^{\infty} t^{\alpha-1} E_{\alpha, \alpha}\left(-a \xi^{2} t^{\alpha}\right) \cos [n(\varphi-\phi)] J_{n}(\rho \xi) J_{n}(r \xi) \xi \mathrm{d} \xi .
$$

In the case of axial symmetry [17],

$$
\mathcal{G}_{Q}(r, \rho, t)=\int_{0}^{\infty} t^{\alpha-1} E_{\alpha, \alpha}\left(-a \xi^{2} t^{\alpha}\right) J_{0}(\rho \xi) J_{0}(r \xi) \xi \mathrm{d} \xi
$$

Dependence of the solution (5.2) on the coordinates $r, \varphi$, and $z$ is depicted in Figures 9, 10 , and 11 with

$$
\overline{\mathcal{G}}_{Q}=\frac{\rho^{3}}{t^{\alpha-1} Q_{0}} \mathcal{G}_{Q}
$$




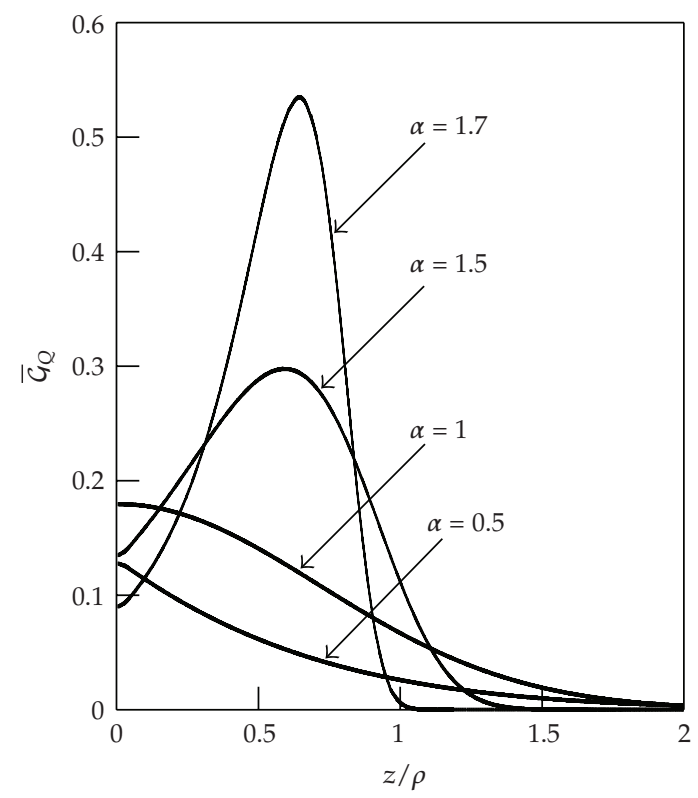

Figure 11: Dependence of nondimensional fundamental solution $\mathcal{G}_{Q}(r, \varphi, z, \rho, \phi, \zeta, t)$ on the spatial coordinate $z$ for $\phi=0, \zeta=0, r=\rho, \varphi=0$, and $\kappa=0.5$.

\section{Discussion}

The solutions to the Cauchy and source problems for time-fractional diffusion-wave equation have been found in cylindrical coordinates. The considered equation in the case $0<\alpha<1$ interpolates the Helmholtz and diffusion equation. In the case $1<\alpha<2$, the time-fractional diffusion-wave equation interpolates the standard diffusion equation and the classical wave equation.

For $1<\alpha<2$, the solutions to the fractional diffusion-wave equation feature propagating humps, underlining the proximity to the standard wave equation in contrast to the shape of curves describing the subdiffusion regime $(0<\alpha<1)$.

For better understanding of behavior of solutions, it is worthwhile to compare the obtained results with those for delta pulse applied at the origin investigated in [32]. The Mittag-Leffler functions arising in (3.5), (4.2), and (5.2) for large values of argument are represented as

$$
\begin{aligned}
E_{\alpha}\left[-a\left(\xi^{2}+\eta^{2}\right) t^{\alpha}\right] & \sim \frac{1}{\Gamma(1-\alpha)} \frac{1}{a\left(\xi^{2}+\eta^{2}\right) t^{\alpha}}, \\
E_{\alpha, 2}\left[-a\left(\xi^{2}+\eta^{2}\right) t^{\alpha}\right] & \sim \frac{1}{\Gamma(2-\alpha)} \frac{1}{a\left(\xi^{2}+\eta^{2}\right) t^{\alpha}}, \\
E_{\alpha, \alpha}\left[-a\left(\xi^{2}+\eta^{2}\right) t^{\alpha}\right] & \sim-\frac{1}{\Gamma(-\alpha)} \frac{1}{\left[a\left(\xi^{2}+\eta^{2}\right) t^{\alpha}\right]^{2}} .
\end{aligned}
$$

Such asymptotic results in singularities of the solution to the first and the second Cauchy problems at the point of application of the delta pulse, whereas the solution to the 
source problem does not have singularity. Dependence of the solution on the angular coordinate $\varphi$ at some distance from the point of the delta pulse application $(r=0.6 \rho$ in Figures 3 and 7) features only humps with no singularity.

\section{References}

[1] F. Mainardi, "Fractional calculus: some basic problems in continuum and statistical mechanics," in Fractals and Fractional Calculus in Continuum Mechanics (Udine, 1996), A. Carpinteri and F. Mainardi, Eds., vol. 378 of CISM Courses and Lectures, pp. 291-348, Springer, Vienna, Austria, 1997.

[2] A. Pȩkalski and K. Sznajd-Weron, Eds., Anomalous Diffusion: From Basics to Applications, Springer, Berlin, Germany, 1999.

[3] R. Metzler and J. Klafter, “The random walk's guide to anomalous diffusion: a fractional dynamics approach," Physics Reports, vol. 339, no. 1, pp. 1-77, 2000.

[4] G. M. Zaslavsky, "Chaos, fractional kinetics, and anomalous transport," Physics Reports, vol. 371, no. 6, pp. 461-580, 2002.

[5] B. J. West, M. Bologna, and P. Grigolini, Physics of Fractal Operators, Institute for Nonlinear Science, Springer, New York, NY, USA, 2003.

[6] R. Metzler and J. Klafter, "The restaurant at the end of the random walk: recent developments in the description of anomalous transport by fractional dynamics," Journal of Physics A, vol. 37, no. 31, pp. R161-R208, 2004.

[7] Y. Z. Povstenko, "Fractional heat conduction equation and associated thermal stress," Journal of Thermal Stresses, vol. 28, no. 1, pp. 83-102, 2005.

[8] R. L. Magin, Fractional Calculus in Bioengineering, Begell House Publishers, Connecticut, Mass, USA, 2006.

[9] V. V. Gafiychuk and B. Yo. Datsko, "Pattern formation in a fractional reaction-diffusion system," Physica A, vol. 365, no. 2, pp. 300-306, 2006.

[10] V. V. Uchaikin, Method of Fractional Derivatives, Artishock, Ulyanovsk, Russia, 2008.

[11] F. Mainardi, "The fundamental solutions for the fractional diffusion-wave equation," Applied Mathematics Letters, vol. 9, no. 6, pp. 23-28, 1996.

[12] W. Wyss, "The fractional diffusion equation," Journal of Mathematical Physics, vol. 27, no. 11, pp. 27822785, 1986.

[13] W. R. Schneider and W. Wyss, "Fractional diffusion and wave equations," Journal of Mathematical Physics, vol. 30, no. 1, pp. 134-144, 1989.

[14] Y. Fujita, "Integrodifferential equation which interpolates the heat equation and the wave equation," Osaka Journal of Mathematics, vol. 27, no. 2, pp. 309-321, 1990.

[15] A. Hanyga, "Multidimensional solutions of time-fractional diffusion-wave equations," The Royal Society of London. Proceedings. Series A, vol. 458, no. 2020, pp. 933-957, 2002.

[16] Y. Z. Povstenko, "Stresses exerted by a source of diffusion in a case of a non-parabolic diffusion equation," International Journal of Engineering Science, vol. 43, no. 11-12, pp. 977-991, 2005.

[17] Y. Z. Povstenko, "Two-dimensional axisymmetric stresses exerted by instantaneous pulses and sources of diffusion in an infinite space in a case of time-fractional diffusion equation," International Journal of Solids and Structures, vol. 44, no. 7-8, pp. 2324-2348, 2007.

[18] B. N. N. Achar and J. W. Hanneken, "Fractional radial diffusion in a cylinder," Journal of Molecular Liquids, vol. 114, no. 1-3, pp. 147-151, 2004.

[19] Y. Z. Povstenko, "Fractional radial diffusion in a cylinder," Journal of Molecular Liquids, vol. 137, no. $1-3$, pp. 46-50, 2008.

[20] N. Özdemir and D. Karadeniz, "Fractional diffusion-wave problem in cylindrical coordinates," Physics Letters A, vol. 372, no. 38, pp. 5968-5972, 2008.

[21] N. Özdemir, O. P. Agrawal, D. Karadeniz, and B. B. Iskender, "Axis-symmetric fractional diffusionwave problem: part I-analysis," in Proceedings of the 6th Euromech Nonlinear Dynamics Conference (ENOC '08), Saint Petrsburg, Russia, June-July 2008.

[22] E. K. Lenzi, L. R. da Silva, A. T. Silva, L. R. Evangelista, and M. K. Lenzi, “Some results for a fractional diffusion equation with radial symmetry in a confined region," Physica A, vol. 388, no. 6, pp. 806-810, 2009.

[23] N. Özdemir, D. Karadeniz, and B. B. Iskender, "Fractional optimal control problem of a distributed system in cylindrical coordinates," Physics Letters A, vol. 373, no. 2, pp. 221-226, 2009. 
[24] N. Özdemir, O. P. Agrawal, D. Karadeniz, and B. B. Iskender, "Fractional optimal control problem of an axis-symmetric diffusion-wave propagation," Physica Scripta T, vol. 136, Article ID 014024, 5 pages, 2009.

[25] X. Jiang and M. Xu, "The time fractional heat conduction equation in the general orthogonal curvilinear coordinate and the cylindrical coordinate systems," Physica A, vol. 389, no. 17, pp. 3368$3374,2010$.

[26] E. K. Lenzi, R. Rossato, M. K. Lenzi, L. R. da Silva, and G. Gonçalves, "Fractional diffusion equation and external forces: solutions in a confined region," Zeitschrift für Naturforschung Section A, vol. 65, no. 5, pp. 423-430, 2010.

[27] H. Qi and J. Liu, “Time-fractional radial diffusion in hollow geometries," Meccanica, vol. 45, no. 4, pp. 577-583, 2010.

[28] R. Gorenflo and F. Mainardi, "Fractional calculus: integral and differential equations of fractional order," in Fractals and Fractional Calculus in Continuum Mechanics (Udine, 1996), A. Carpinteri and F. Mainardi, Eds., vol. 378 of CISM Courses and Lectures, pp. 223-276, Springer, Vienna, Austria, 1997.

[29] A. A. Kilbas, H. M. Srivastava, and J. J. Trujillo, Theory and Applications of Fractional Differential Equations, vol. 204 of North-Holland Mathematics Studies, Elsevier Science B.V., Amsterdam, The Netherlands, 2006.

[30] I. Podlubny, Fractional Differential Equations, vol. 198 of Mathematics in Science and Engineering, Academic Press, San Diego, Calif, USA, 1999.

[31] A. Erdélyi, W. Magnus, F. Oberhettinger, and F. G. Tricomi, Higher Transcendental Functions. Vol. III, McGraw-Hill, New York, NY, USA, 1955.

[32] Y. Z. Povstenko, "Fundamental solutions to three-dimensional diffusion-wave equation and associated diffusive stresses," Chaos, Solitons E Fractals, vol. 36, no. 4, pp. 961-972, 2008. 